\title{
When Horses and Zebras Coexist: Achieving Diagnostic Excellence in the Age of High-Value Care
}

\author{
Brett G Fischer, MD*, Andrew PJ Olson, MD
}

Weill Department of Medicine, Weill Cornell Medicine; Departments of Medicine and Pediatrics, University of Minnesota Medical School, Minneapolis, Minnesota.

afe, timely, and efficient diagnosis is fundamental for high-quality, effective healthcare. Why is diagnosis so important? First, it informs the two other main areas of medical decision-making: treatment and prognosis. These are the means by which physicians can actually change health outcomes for patients, as well as ensure that patients and their families have a realistic and accurate understanding of what the future holds with respect to their health. Second, patients and families tend to feel a sense of closure from having a name and an explanation for symptoms, even in the absence of specific treatment. Proper labeling allows patients and families to connect with others with the same diagnosis, who are best positioned to offer empathy by virtue of their similar experiences.

Despite the fundamental role of diagnosis, diagnostic error is pervasive in medicine, with unacceptable levels of resultant harm. ${ }^{1}$ In 2015, the Institute of Medicine published a landmark report, "Improving Diagnosis in Health Care," bringing the problem to the forefront of the minds of healthcare professionals and the general public alike. According to the report, "improving the diagnostic process...represents a moral, professional, and public health imperative." ${ }^{\prime 1}$ We must do more than avoid diagnostic error, however-we must aim to achieve diagnostic excellence. Not getting it wrong is not enough.

There are real challenges to achieving diagnostic safety, let alone excellence. The "churn" of modern hospital medicine does not reward deep diagnostic thought, nor does it often encourage reflection or collaboration, important components of being able to achieve diagnostic excellence. ${ }^{2}$ Furthermore, despite their years of training, physicians often have difficulty applying probabilistic reasoning and appropriately incorporating diagnostic information in the best evidence-based manner. ${ }^{3,4}$ In addition, there are no validated measures of diagnostic performance in practice. It is telling that many hospitalists, despite a professed interest in complex diagnosis, would rather be assigned to care for a patient with cellulitis than a patient with a complicated differential diagnosis.

Given these challenges, how can the modern healthcare ecosystem be changed to achieve diagnostic excellence? In this month's issue of Journal of Hospital Medicine, Singer and colleagues describe a pilot project of a proposed solution to

Corresponding Author: Brett Fischer; E-mail: Brf9036@med.cornell.edu; Telephone: 212-746-4071

Received: November 6, 2019; Accepted: November 7, 2019

๑ 2020 Society of Hospital Medicine DOI 10.12788/jhm.3356 the problem. ${ }^{5}$ Aptly named, the Socrates Project is an intervention that makes available a team of "diagnosticians" that can be consulted for assistance with challenging diagnostic cases. The physicians on the team volunteer their time, allowing for deep diagnostic evaluation that is not limited by one's daily workload, thus overcoming one of the major hurdles to achieving diagnostic excellence. The described program also focuses on harnessing the power of teamwork, which is especially relevant given recent descriptions of the effectiveness of collective intelligence in improving diagnostic performance. ${ }^{6} \mathrm{Im}$ portantly, the authors recognize that their intervention will not achieve a diagnosis in every case for which they are consulted; rather, they hope that their thorough evaluation will uncover additional potential diagnostic avenues for the referring team to pursue, with a goal to "improve patient care by providing... ideas to reduce —or at least manage—diagnostic uncertainty."

Programs of this nature are exciting for hospitalists. Hospital medicine is, perhaps, a place in modern medicine where diagnostic excellence has a natural home. Patients admitted to the hospital are acutely (and often severely) ill, and hospitalists are tasked with rapidly identifying the cause of their illness in order to initiate appropriate treatment and accurately inform prognosis. Hospitalists, as generalists, take a broad approach to challenging cases, and they tend to practice in well-resourced environments with nearly every diagnostic modality at their disposal. Many hospitalists would envy participating in a program such as the Socrates Project.

While Singer et al.'s innovation-and the institutional support thereof-should be lauded, some discussion must be had about how to assess the effectiveness of such a program. The authors acknowledge the need for evaluation of both the diagnostic process and the outcomes that process achieves. Measuring diagnostic performance is challenging, however, and while there is substantial progress being made in this area, recent efforts tend to focus on identifying diagnostic errors rather than measuring diagnostic excellence. Moreover, even if a program does improve diagnostic performance, how should we evaluate for unintended consequences of its implementation? In the age of high-value care, how can we ensure that efforts to do a better job of spotting proverbial zebras do not come at the cost of harming too many horses?

Hospitalists are well primed to answer this question. The juxtaposition of Singer et al.'s article with the Journal of Hospital Medicine's long-running series on Choosing Wisely®: Things We Do for No Reason ${ }^{\mathrm{TM}}$ provides a natural synergy to begin crafting a framework to evaluate unintended consequences of 
a program in diagnostic excellence. More diagnosis is not the goal; more appropriate diagnosis is what is needed. A clinical program aimed at achieving diagnostic excellence should not employ low-value, wasteful strategies that do not add substantively to the diagnostic process but should instead seek to improve the overall efficiency of even complicated diagnostic odysseys. Avoiding waste throughout will allow for allocation of diagnostic resources where they are needed. In turn, hospitalists can do a better job of correctly identifying both horses and zebras for what they are. While a given hospitalization for a diagnostically complex patient may be relatively expensive, better diagnosis during an index hospitalization is likely to lead to decreased downstream costs, such as those related to readmissions and further testing, as well as better health outcomes.

The Socrates Project, along with similar programs at other institutions, are exciting innovations. These programs are not only likely to be good for patients but are also good for hospitalists. The field of hospital medicine should leverage its collective expertise in clinical medicine, systems of care, and high-value care to become a home for diagnostic excellence.
Disclosures: The authors have nothing to disclose.

\section{References}

1. National Academies of Sciences, Engineering, and Medicine. Improving Diagnosis in Health Care. Washington, DC: The National Academies Press: 2015. https://doi.org/10.17226/21794

2. Olson A, Rencic J, Cosby K, et al. Competencies for improving diagnosis: an interprofessional framework for education and training in health care. Diagnosis. 2019;6(4):335-341. https://doi.org/10.1515/dx-2018-0107.

3. Baduashvili A, Guyatt G, Evans AT. ROC anatomy - getting the most out of your diagnostic test. J Gen Intern Med. 2019;34(9):1892-1898. https://doi. org/10.1007/s11606-019-05125-0.

4. Manrai AK, Bhatia G, Strymish J, Kohane IS, Jain SH. Medicine's uncomfortable relationship with math: calculating positive predictive value. JAMA Intern Med. 2014;174(6):991-993. https://doi.org/10.1001/jamainternmed.2014.1059.

5. Singer BD, Goodwin AM, Patel AA, Vaughan DE. The Socrates Project for difficult diagnosis at Northwestern Medicine. J Hosp Med. 2020;15(2):116118. https://doi.org/ 10.12788/jhm.3335.

6. Barnett ML, Boddupalli D, Nundy S, Bates DW. Comparative accuracy of diagnosis by collective intelligence of multiple physicians vs individual physicians. JAMA Netw Open. 2019;2(3):e190096. https://doi.org/10.1001/jamanetworkopen.2019.0096.

7. Dhaliwal G. Bringing high-value care to the inpatient teaching service. JAMA Intern Med. 2014;174(7):1021-1022. https://doi.org/10.1001/jamainternmed.2014.2012. 